\title{
Intravenous immunoglobulin for the treatment of autoimmune encephalopathy in children with autism
}

\author{
Kathleen Connery ${ }^{1}$, Marie Tippett ${ }^{1}$, Leanna M. Delhey ${ }^{1}$, Shannon Rose $\mathbb{0}^{1}$, John C. Slattery ${ }^{2}$, Stephen G. Kahler ${ }^{1}$, \\ Juergen Hahn $\mathbb{1}^{3,4}$, Uwe Kruger ${ }^{3}$, Madeleine W. Cunningham ${ }^{5}$, Craig Shimasaki ${ }^{6}$ and Richard E. Frye $\mathbb{B}^{7,8}$
}

\begin{abstract}
The identification of brain-targeted autoantibodies in children with autism spectrum disorder (ASD) raises the possibility of autoimmune encephalopathy (AIE). Intravenous immunoglobulin (IVIG) is effective for AIE and for some children with ASD. Here, we present the largest case series of children with ASD treated with IVIG. Through an ASD clinic, we screened 82 children for AIE, 80 of them with ASD. IVIG was recommended for 49 (60\%) with 31 (38\%) receiving the treatment under our care team. The majority of parents (90\%) reported some improvement with $71 \%$ reporting improvements in two or more symptoms. In a subset of patients, Aberrant Behavior Checklist (ABC) and/or Social Responsiveness Scale (SRS) were completed before and during IVIG treatment. Statistically significant improvement occurred in the SRS and ABC. The antidopamine D2L receptor antibody, the anti-tubulin antibody and the ratio of the antidopamine $D 2 L$ to $D 1$ receptor antibodies were related to changes in the $A B C$. The Cunningham Panel predicted SRS, ABC, parent-based treatment responses with good accuracy. Adverse effects were common (62\%) but mostly limited to the infusion period. Only two (6\%) patients discontinued IVIG because of adverse effects. Overall, our open-label case series provides support for the possibility that some children with ASD may benefit from IVIG. Given that adverse effects are not uncommon, IVIG treatment needs to be considered cautiously. We identified immune biomarkers in select IVIG responders but larger cohorts are needed to study immune biomarkers in more detail. Our small open-label exploratory trial provides evidence supporting a neuroimmune subgroup in patients with ASD.
\end{abstract}

\section{Background}

Autism spectrum disorder (ASD) is a behaviorally defined disorder, which now affects $\sim 2 \%$ of children in the United States ${ }^{1}$. Although the standard-of-care for ASD is behavioral therapy, such therapy requires full-time engagement with one or several therapists for many years. In many cases, outcomes are suboptimal and/or incomplete $^{2}$. Thus, medical therapies that can augment behavioral therapy are urgently needed.

Correspondence: Richard E. Frye (rfrye@phoenixchildrens.com)

'University of Arkansas for Medical Sciences, Little Rock, AR 77205, USA

${ }^{2}$ BioROSA Technologies Inc, San Francisco, CA 94103, USA

Full list of author information is available at the end of the article.
Recent studies suggest that ASD is associated with a variety of physiological abnormalities including immune system dysfunction ${ }^{3}$. For example, a maternal immune response induced during gestation results in ASD-like behavior in offspring in the maternal immune activation (MIA) rodent ${ }^{4,5}$ and replacing the immune system using bone marrow transplant corrected many symptoms in a mouse model of Rett syndrome, a syndrome closely aligned with $\mathrm{ASD}^{6}$. In individuals with ASD, inflammatory cytokines are elevated in the blood ${ }^{7}$ and brain $^{8}$ and microglia are active in the brain $^{8}$. Children with ASD have autoantibodies to brain tissue such as myelin basic protein, serotonin receptors, brain endothelium, cerebellar tissue, and glutamic acid decarboxylase (GAD) as well as

\section{(c) The Author(s) 2018}

(c) Open Access This article is licensed under a Creative Commons Attribution 4.0 International License, which permits use, sharing, adaptation, distribution and reproduction cc) in any medium or format, as long as you give appropriate credit to the original author(s) and the source, provide a link to the Creative Commons license, and indicate if changes were made. The images or other third party material in this article are included in the article's Creative Commons license, unless indicated otherwise in a credit line to the material. If material is not included in the article's Creative Commons license and your intended use is not permitted by statutory regulation or exceeds the permitted use, you will need to obtain permission directly from the copyright holder. To view a copy of this license, visit http://creativecommons.org/licenses/by/4.0/. 
to non-brain tissue such as the folate receptor alpha $(\mathrm{FR} \alpha)^{9}$ and mitochondria ${ }^{3}$. Children with autoantibodies are reported to have a more severe form of $\mathrm{ASD}^{10-15}$. The role of autoantibodies during gestation is exemplified by maternal antibodies to fetal brain that have high specificity for the development of ASD in the offspring ${ }^{16}$ with a relatively more severe phenotype ${ }^{17}$ and brain enlargement ${ }^{18}$.

The overlap between ASD and Pediatric Autoimmune Neuropsychiatric Disorders Associated with Streptococcal Infection (PANDAS) and Pediatric Acute-Onset Neuropsychiatric Syndrome (PANS) has been undergoing study by Cunningham et al. (in preparation) over the last several years. PANDAS/PANS-associated autoantibodies accelerate the function of the calcium calmodulindependent protein kinase II (CaMKII) enzyme, a multifunctional enzyme highly concentrated in the brain that controls neurotransmission and neuronal excitability ${ }^{19}$ and regulates catecholamine ${ }^{20}$ and glutamate ${ }^{21}$ neurotransmission. Abnormalities in CaMKII function has been linked to movement and neuropsychiatric disorders in children $^{22,23}$ and genetic abnormalities in the CaMKII genes are linked to intellectual disability ${ }^{24}$. Furthermore, previous studies have linked PANDAS/PANS-associated biomarkers with behaviors commonly seen in ASD. For example, high levels of either the anti-lysoganglioside GM1 antibody or CaMKII are associated with tics and obsessive-compulsive disorder behavior ${ }^{25}$ and high antidopamine D2L to D1 antibody ratio is associated with irritability, a frequently targeted ASD symptom ${ }^{26}$.

Brain-targeted autoantibodies in an individual with central nervous system dysfunction point toward a diagnosis of an autoimmune encephalopathy (AIE). In children and adolescents AIE is most commonly diagnosed as non-neoplastic limbic encephalitis, resulting from $\mathrm{GAD}^{27-33}$, voltage-gated potassium channel (VGKC) $)^{29,34}$, or $N$-methyl-D-aspartate (NMDA) receptor ${ }^{34-38}$ autoantibodies or without autoantibodies ${ }^{28-30,34,39}$. Seizure is the most common presenting symptom but children commonly also manifest motor, cognitive, behavioral, and/or psychiatric symptoms including aphasia ${ }^{35}$, declining academic functioning ${ }^{27}$, depression ${ }^{29}$, anxiety ${ }^{29}$, amnesia $^{29,34,35}$, learning problems ${ }^{29}$, loss of social skills ${ }^{38}$, hallucinations $^{34}$, agitation ${ }^{34,39}$, mood disorder ${ }^{34}$, behavioral changes $\mathrm{s}^{30,35,39}$, decreased appetite ${ }^{36}$, irritability ${ }^{36}$, dysautonomia $^{30,34,39}$, movement disorder ${ }^{35}$, peripheral neuropathy $^{32}$, dystonia ${ }^{33}$, and insomnia ${ }^{36,39}$. Both GAD $65^{40}$ and NMDA receptor autoantibodies ${ }^{36-38}$ have been associated with ASD with the NMDA receptor autoantibody being associated with regressive $e^{36,38}$ and late-onset ${ }^{37}$ ASD.

Case series ${ }^{28-30,34,35}$ and case reports ${ }^{27,31-33,36,39}$ confirm that Intravenous immunoglobulin (IVIG) is useful for AIE in children and adolescents. IVIG has been shown to improve symptoms of ASD, including aberrant behavior $^{41}$, speech and social interaction ${ }^{42}$, and ASD-related behaviors ${ }^{43}$. A small double-blind placebo-controlled study reported significant improvements in children with ASD receiving IVIG on the Aberrant Behavior Checklist $(\mathrm{ABC})$ compared with those receiving placebo ${ }^{44}$. Another study demonstrated neurodevelopmental regression when discontinuing IVIG treatment ${ }^{41}$. In contrast, smaller case series did not find improvements in children with ASD with IVIG treatment ${ }^{45,46}$. As IVIG has been used to treat PANDAS/PANS symptoms in a placebo-controlled study $^{47}$, case reports ${ }^{48}$, and case series ${ }^{49}$, IVIG could be a treatment for children with ASD with PANDAS/PANS symptoms.

Given that children with ASD may manifest brain autoantibodies, we screened patients in an ASD clinic who did not respond to standard interventions for AIE. Selected patients with possible AIE were offered IVIG as a potential treatment. As part of our clinical protocol, families were asked to complete specific behavioral questionnaires, allowing the examination of the change in behavioral ratings for patients that received IVIG. In addition, clinical notes were analyzed to determine symptoms improvement, adverse effect, and treatment course. We also examined if immune biomarkers could predict response to IVIG treatment. Lastly, we provide several cases as illustrations of the treatment course with IVIG. This report provides insight to the possible utility of IVIG treatment in children with ASD as well as the potential limitations of this treatment.

\section{Methods}

The Institutional Review Board at the University of Arkansas for Medical Sciences (Little Rock, AR) approved the study. Parents of participants provided written informed consent. The study is registered as NCT02003170 on clinicaltrials.gov.

All patients evaluated in our ASD multispecialty clinic for AIE were included in this study. The majority of the patients (80/82) were diagnosis with ASD, except for two females, one who was a sibling of a male with ASD and AIE who was treated for severe atypical learning disabilities and attention deficit and one with severe tics and behavioral dysregulation.

ASD was diagnosed based upon one of the following criteria: (i) a gold-standard diagnostic instrument such as the Autism Diagnostic Observation Schedule and/or Autism Diagnostic Interview-Revised; (ii) the state of Arkansas diagnostic standard, defined as agreement of a physician, psychologist and speech therapist; and/or (iii) Diagnostic and Statistical Manual of Mental Disorders (DSM) diagnosis by a physician along with standardized validated questionnaires and diagnosis confirmation by the Principal Investigator (R.E.F.). We have validated that 
this criteria captures an accurate diagnosis of ASD in our previous studies by re-evaluating a portion of the participants with the ADI- $\mathrm{R}^{50-53}$.

Table S1 provides participant characteristics. Comorbid conditions were derived from a parent-reported medical questionnaire and from review of medical records. Regression (defined as loss of already obtained skills) was defined in detail in our questionnaire. Questions regarding regression included the timing, specific skills lost, duration of the regression, trigger (if known), and whether or not there were multiple regressions or a single regression. This method for assessing medical comorbidities has been used in several of our previous studies ${ }^{50-}$ 53.

\section{Immune markers measured}

The evaluation for AIE focused on detecting brain autoantibodies as measured by several panels. Paraneoplastic panel (PNP; Mayo Medical Laboratories, Rochester, $\mathrm{MN}$ ) measures antineuronal nuclear, anti-glial nuclear, Purkinje cytoplasmic, $\mathrm{P} / \mathrm{Q}$ and N-type calcium, and VGKC antibodies as well as GAD65, AMPA, NMDA, and GABA-B receptor antibodies. Because GAD $65^{40}$ and NMDA receptor ${ }^{36-38,54}$ autoantibodies have been associated with ASD, these two autoantibodies were measured specifically in most patients. The Cunningham panel (antidopamine D1 receptor (D1R), antidopamine D2L receptor (D2R), anti-lysoganglioside GM1, anti-tubulin, CaMKII; Moleculera Lab Inc, Oklahoma City, OK) was also performed as the symptoms associated with these autoantibodies overlap with behavioral symptoms of ASD. In some patients, the FR $\alpha$ autoantibody was measured because of its close association with $\mathrm{ASD}^{9,51,52,55}$. Some patients also had tests performed for markers of immune activation such as $\mathrm{C} 3, \mathrm{C} 4, \mathrm{CH} 50, \mathrm{C}$-reactive protein, and erythrocyte sedimentation rate. Using findings from these laboratory tests along with clinical symptoms, the need for treatment of AIE with IVIG was considered, which is the focus of this report.

\section{Direct enzyme-linked immunosorbent assay (ELISA)}

Ninety-six-well microtiter plates (Greiner Bio-One, Monroe, NC) were coated with $50 \mu \mathrm{L}$ of antigen in $100 \mathrm{mM}$ carbonate/bicarbonate buffer (pH 9.6) and stored up to 2 weeks at $4{ }^{\circ} \mathrm{C}$. Antigen coating concentrations were as follows: $10 \mu \mathrm{g} / \mathrm{mL}$ of purified tubulin (MP Biomedicals, Santa Ana, CA), $10 \mu \mathrm{g} / \mathrm{mL}$ of dopamine D1 receptor (D1R, Perkin Elmer, Waltham, MA), $10 \mu \mathrm{g} / \mathrm{mL}$ dopamine D2L receptor (D2R, Perkin Elmer), and $20 \mu \mathrm{g} /$ $\mathrm{mL}$ of lysoganglioside $\mathrm{G}_{\mathrm{M} 1}$ (Sigma Aldrich, Darmstadt, Germany). Tubulin-, D1R-, and D2R-coated plates were washed three times with phosphate-buffered saline (PBS, $\mathrm{pH}$ 7.2) containing $0.1 \%$ Tween (ThermoFisher Scientific, Waltham, MA). Lysoganglioside-coated plates were washed three times in PBS without Tween in all steps. Plates were blocked with $1 \%$ bovine serum albumin (ThermoFisher Scientific) in PBS for 60 minutes at $37^{\circ} \mathrm{C}$. Serum or cerebrospinal fluid (CSF) samples serially diluted in 1\% BSA (in PBS) were added to washed plates, then incubated overnight at $4{ }^{\circ} \mathrm{C}$. The next day, plates were washed as described above and primary IgG antibody binding was detected by adding $50 \mu \mathrm{L}$ per well of diluted alkaline phosphatase-conjugated goat anti-human $y$ chain-specific secondary antibody (polyclonal, Cat\# A3312, Sigma Aldrich) and incubated for $60 \mathrm{~min}$ at $37^{\circ} \mathrm{C}$. Final dilution of secondary antibody was determined empirically for each antigen and validated for every new antibody lot. Plates were developed at $26^{\circ} \mathrm{C}$ for $2 \mathrm{~h} \pm$ $15 \mathrm{~min}$ with $50 \mu \mathrm{L}$ per well of $1 \mathrm{mg} / \mathrm{mL} p$-nitrophenylphosphatase (Sigma Aldrich) in $0.1 \mathrm{M}$ diethanolamine buffer ( $\mathrm{pH}$ 9.8). Optical density values were measured at $405 \mathrm{~nm}$ on an automated BioTek microplate reader (BioTek Instruments, Winooski, VT) and corrected by blanks (wells coated with antigen, without serum added). All samples were assayed in duplicate and averaged. Duplicates not matching with $<20 \%$ variance were repeated. Titers represent the serum dilution at optical density of 0.1 at $405 \mathrm{~nm}$ after $2 \mathrm{~h}$. Known positive and negative control samples were included on each plate to standardize and monitor assay performance ${ }^{20,29}$. Each new lot of all reagents and antibodies were validated using serum samples with known titers.

\section{Cell culture}

SK-N-SH human neuroblastoma cells obtained from American Type Culture Collection (HTB-11, Manassas, VA) were grown in complete F12-Dulbecco's Modified Eagle Medium (ThermoFisher Scientific) as previously described $^{15}$. Complete media contained $10 \%$ fetal bovine serum (ThermoFisher Scientific) and $1 \%$ penicillin-streptomycin antibiotic (ThermoFisher Scientific). Cellular extracts used in the CaMKII assay were centrifuged at $15,000 \mathrm{rpm}$ for $10 \mathrm{~min}$ at $4{ }^{\circ} \mathrm{C}$. Protein concentrations of the extracts were determined by Bradford assay using the Protein Assay Kit II (Bio-Rad, Hercules, CA).

\section{CaMKII activity assay}

Assay for CaMKII activity was performed as previously described $^{15}$. In brief, SK-N-SH cells were plated in sixwell plates at 2.5 million cells/well and incubated overnight in complete F12-DMEM, at $37^{\circ} \mathrm{C}$ with $5 \% \mathrm{CO} 2$. The next day, cells were serum starved for 30 minutes in serum-free $\mathrm{F} 12$ media with $2 \mathrm{mM} \mathrm{CaCl} 2,2 \mathrm{mM} \mathrm{KCl}$, and $0.4 \mathrm{mM} \mathrm{MgCl} 2$, then stimulated for 30 minutes with patient sera or CSF diluted 1:100 in the same media or with media alone (basal control). Cells were harvested, centrifuged, solubilized in $0.165 \mathrm{~mL}$ of protein extraction 
buffer with protease inhibitors (Soybean Trypsin Inhibitor, PMSF, Leupeptin, and Aprotinin, Sigma Aldrich, St. Louis, MO), and homogenized. Enzymatic activity was measured using the CaMKII assay system (Promega, Madison, WI) per manufacturer's instructions. In brief, $5 \mu \mathrm{L}$ of cell lysate was incubated with $50 \mu \mathrm{M}$ peptide substrate, buffers and ATP $\left[\gamma^{-32} \mathrm{P}\right]$ (Perkin Elmer) for 2 minutes at $30^{\circ} \mathrm{C}$. Samples were spotted onto capture membranes and washed. Radioactivity retained on the membrane was measured by scintillation counter (Beckman Coulter, Indianapolis, IN) and used to calculate specific activity of the CaMKII enzyme $(\mathrm{pmoL} / \mathrm{min} / \mu \mathrm{g})$ as described in kit instructions. The protein concentration of each sample was used to standardize the CaMKII enzyme activity, and the percentage of specific activity of baseline (basal control) was calculated for each sample where the basal level was set at $100 \%$. All samples were assayed in triplicate and results were averaged. Sera from patients with known high and low CaMKII activity and a basal control sample were included to standardize the assay.

\section{Parent-reported clinical outcomes}

The first author, who was not involved in patient care, performed a qualitative-quantitative analysis of clinical notes. The senior author (R.E.F.), the primary treating physician, confirmed this analysis for accuracy. The notes were analyzed for specific symptoms mentioned in regards to improvements and worsening. Areas of improvement were quantified if they occurred in $\geq 10 \%$ of the participants. Symptom improvement was classified into eight non-exclusive categories (See Table 2), "other" and "no improvement." The frequency of these reported improvements across patients was calculated and the total number of improved symptoms was also analyzed for each patient. A similar chart review was performed for determining frequency of adverse effects, although there was no lower limit for reporting the frequency of adverse effects.

\section{Cognitive and behavioral outcome measures}

The $\mathrm{ABC}$ is a 58 -item questionnaire ${ }^{56}$ that measures disruptive behaviors and has convergent and divergent validity $^{57}$. The Social Responsiveness Scale (SRS) is a 65item questionnaire that measures the severity of social skill deficits across five domains ${ }^{58}$, which has good concordance with the $\mathrm{ADOS}^{59}$. Caretakers are asked to complete these questionnaires prior to each clinical visit to assist in the evaluation of the change in symptoms at each visit. One important caveat is that caretakers were not required to fill out these questionnaires as declining did not ethically prevent their child from appropriate medical care.

\section{Statistical analysis}

Statistical analyses were performed with SAS 9.4 (SAS Institute Inc., Cary, NC) and MATLAB (The Mathworks, Natick, MA). Graphs were produced using Excel version 14.0 (Microsoft Corp, Redmond, WA). To determine whether any significant change occurred in behavior questionnaires with treatment an analysis of variance implemented as a mixed-model regression was utilized. The models included random effect of time (before/during treatment) and intercept to account for individual symptom level. The models tested the a priori hypothesis that a significant change in the outcome measure occurred with treatment and used a $\alpha \leq 0.05$. All of the available questionnaire data were used for each participant. There was no imputation for missing data. Participants without questionnaire data both before and after the start of treatment were excluded. The Cohen's $d$ ' effect size was calculated for each statistical comparison.

We sought to evaluate whether biomarkers of autoimmunity could predict treatment response. As the Cunningham panel was the only frequently abnormal panel, only the Cunningham panel was used to predict treatment response. We examined the relationship between the change in questionnaire score with IVIG treatment and the baseline components of the Cunningham panel. Only the total scores for the ABC and SRS questionnaires were examined to reduce excessive statistical comparisons. However, we also examined the relationship between the Anti-Dopamine D2L/D1 titer ratio and irritability subscale of the $\mathrm{ABC}$, as this relationship was previously reported ${ }^{26}$. We applied a logarithmic transformation with a base of 2 to the autoantibody titers as they increase in exponential steps. As many individuals completed multiple questionnaires both before and during the treatment period, we calculated the average total score of all questionnaires during treatment minus the average total score prior to treatment for each individual as a difference score. We then calculated the correlation between this difference score vs immune biomarkers.

We then defined responders by examining the histogram of the change in the ABC and SRS scores to determine the distribution of responders. As the scale of the Cunningham panel components are quite different, in order to conduct calculations on the same scale across all components, as is standard is statistical linear models, were mean-centered at zero and normalized to have a standard deviation of unity. We then determined whether the components of the Cunningham panel could be used to predict which participants were responders and which where non-responders using Fisher Discriminant Analysis in MATLAB using in-house developed routine. Discriminant analysis creates a linear function that uses the input component (i.e., components of the Cunningham 
panel) to calculate a score that represent whether a particular participant is more like a responder or nonresponses. The statistical distribution of these Fisher Discriminant Analysis scores was estimated via Kernel Density Estimation and a threshold was determined which could optimally divide the scores calculated by the linear discriminant function for the participants into responders and non-responders (i.e., a binary classifier). We report the accuracy, sensitivity, and specificity of the discriminant function.

\section{Results}

\section{Characterization of AIE}

NMDA receptor autoantibodies were negative in all of the 34 patients in which it was tested. GAD65 titer elevation was found in three $(5 \% ; 13.00,0.11,0.07$; normal $(\mathrm{nl})<0.02)$ of the 60 patients in whom it was tested. The PNP was positive for four (6\%) of the 63 patients in which it was tested. For the PNP, two of the cases had VGCC autoantibodies (N-type 0.13; $\mathrm{nl}<0.03$; P/Q-type 0.05, $\mathrm{nl}<$ $0.02)$ and two had striated muscle autoantibodies (1:960 and 1:1920, $\mathrm{nl}<1: 120$ ).

The Cunningham panel was considered to be is positive only when one of the four autoantibody titers was positive in the ELISA AND at the same time autoantibodymediated CaMKII activation was positive ( $>130$ above the basal rate of CaMKII in SKNSH human neuronal cell lines as previously described ${ }^{22}$ ). In this way, the CaMKII elevation was used as a functional confirmation of the consequence of elevated autoantibodies in the ELISA. In the ELISA, the antineuronal autoantibodies were positive when anti-tubulin was $\geq 2000$ titer, anti-lysoganglioside was $\geq 640$ titer, anti-D1R was $\geq 4000$ titer, and anti-D2R was $\geq 16,000$ titer. Endpoint titers were determined at 0.092 cutoff and the titers were determined by routine ELISA as described above.

Using our criterion, the Cunningham panel was positive in $44(57 \%)$ of the 77 patients in which it was performed. In $67 \%$ of the 33 cases, the Cunningham panel was negative because of a normal CaMKII, whereas it was negative because of normal ELISA autoantibody titers in $30 \%$ of the cases and was normal for both ELISA autoantibodies and CaMKII in only one case.

In addition to the aforementioned tests, patients with severe behavioral or medical symptoms including drugresistance epilepsy and/or immunodeficiency were considered candidates for IVIG treatment. If the patient was previously non-responsive to IVIG treatment, alternative treatments were considered. Also, consideration was given to whether other treatments should be tried as an alternative to IVIG. The severity of the ASD and associated behavioral and other symptoms were a determinant when deciding whether a trial of IVIG was warranted.

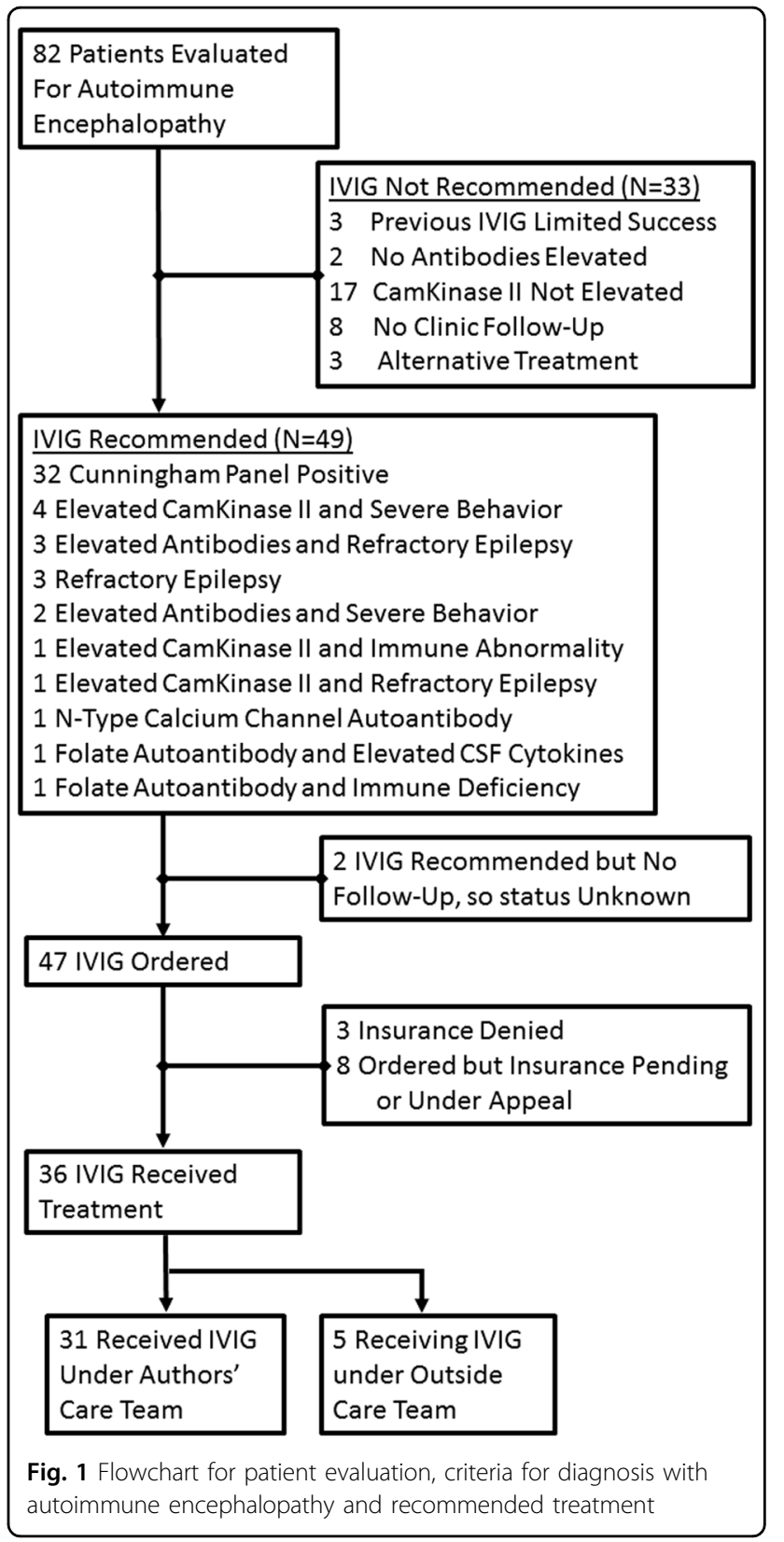

A trial of IVIG was recommended in $49(60 \%)$ of the patients evaluated (See Fig. 1). Thirty-six (73\%) of the patients in whom it was recommended received the treatment with five receiving the treatment by another care team. We reviewed the response to IVIG for patients under our care.

\section{Intravenous immunoglobulin treatment}

Table 1 outlines the starting and current (or ending) dosing for all patients. The most common dose initiated was $2 \mathrm{~g} / \mathrm{kg}$ monthly with the most common dosing $1 \mathrm{~g} / \mathrm{kg} /$ 
Table 1 Starting and Most Recent Dosing Schedule for Intravenous Immunoglobulin (IVIG) Treatment

\begin{tabular}{|c|c|c|c|c|c|}
\hline Starting dosing schedule & Monthly dose & Number of patients & Most recent dosing schedule ${ }^{a}$ & Monthly dose & Number of patients \\
\hline $2.0 \mathrm{~g} / \mathrm{kg} \times 1$ days monthly & $2.0 \mathrm{~g} / \mathrm{kg}$ & $10 \%(3 / 31)$ & $1.0 \mathrm{~g} / \mathrm{kg} \times 2$ days every $3 \mathrm{wks}$ & $2.7 \mathrm{~g} / \mathrm{kg}$ & $6 \%(2 / 30)$ \\
\hline $1.0 \mathrm{~g} / \mathrm{kg} \times 2$ days monthly & $2.0 \mathrm{~g} / \mathrm{kg}$ & $74 \%(23 / 31)$ & $0.8 \mathrm{~g} / \mathrm{kg} \times 3$ days monthly & $2.4 \mathrm{~g} / \mathrm{kg}$ & $3 \%(1 / 30)$ \\
\hline $0.75 \mathrm{~g} / \mathrm{kg} \times 2$ days monthly & $1.5 \mathrm{~g} / \mathrm{kg}$ & $3 \%(1 / 31)$ & $2.0 \mathrm{~g} / \mathrm{kg} \times 1$ days monthly & $2.0 \mathrm{~g} / \mathrm{kg}$ & $10 \%(3 / 30)$ \\
\hline $1.0 \mathrm{~g} / \mathrm{kg} \times 1$ days monthly & $1.0 \mathrm{~g} / \mathrm{kg}$ & $3 \%(1 / 31)$ & $1.0 \mathrm{~g} / \mathrm{kg} \times 2$ days monthly & $2.0 \mathrm{~g} / \mathrm{kg}$ & $37 \%(11 / 30)$ \\
\hline $0.8 \mathrm{~g} / \mathrm{kg} \times 1$ days monthly & $0.8 \mathrm{~g} / \mathrm{kg}$ & $7 \%(2 / 31)$ & $1.0 \mathrm{~g} / \mathrm{kg} \times 1$ days every $2 \mathrm{wks}$ & $2.0 \mathrm{~g} / \mathrm{kg}$ & $3 \%(1 / 30)$ \\
\hline \multirow[t]{9}{*}{$1.0 \mathrm{~g} / \mathrm{kg} \times 2$ days once } & & $3 \%(1 / 31)$ & $0.8 \mathrm{~g} / \mathrm{kg} \times 1$ days every $2 \mathrm{wks}$ & $1.6 \mathrm{~g} / \mathrm{kg}$ & $3 \%(1 / 30)$ \\
\hline & & & $0.75 \mathrm{~g} / \mathrm{kg} \times 2$ days monthly & $1.5 \mathrm{~g} / \mathrm{kg}$ & $3 \%(1 / 30)$ \\
\hline & & & $2.0 \mathrm{~g} / \mathrm{kg} \times 1$ day every & $1.3 \mathrm{~g} / \mathrm{kg}$ & $3 \%(1 / 30)$ \\
\hline & & & $1.0 \mathrm{~g} / \mathrm{kg} \times 1$ day every $3 \mathrm{wks}$ & $1.3 \mathrm{~g} / \mathrm{kg}$ & $10 \%(3 / 30)$ \\
\hline & & & $1.3 \mathrm{~g} / \mathrm{kg} \times 1$ day monthly & $1.3 \mathrm{~g} / \mathrm{kg}$ & $3 \%(1 / 30)$ \\
\hline & & & $1.0 \mathrm{~g} / \mathrm{kg} \times 1$ day monthly & $1.0 \mathrm{~g} / \mathrm{kg}$ & $3 \%(1 / 30)$ \\
\hline & & & $0.8 \mathrm{~g} / \mathrm{kg} \times 1$ day monthly & $0.8 \mathrm{~g} / \mathrm{kg}$ & $6 \%(2 / 30)$ \\
\hline & & & $1.0 \mathrm{~g} / \mathrm{kg} \times 1$ day every $6 \mathrm{wks}$ & $0.7 \mathrm{~g} / \mathrm{kg}$ & $3 \%(1 / 30)$ \\
\hline & & & $0.4 \mathrm{~g} / \mathrm{kg} \times 1$ day monthly & $0.4 \mathrm{~g} / \mathrm{kg}$ & $3 \%(1 / 30)$ \\
\hline
\end{tabular}

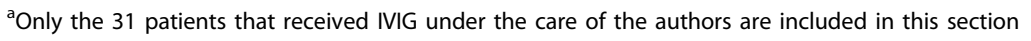

day for 2 days monthly. Of the 31 patients, the dose and/ or frequency of treatment was adjusted in 13 (42\%) to provide an optimal effect. The dose may have been decreased to minimize adverse effects, whereas the treatment interval may have been increased or decreased if the effect appeared to last longer or shorter than the original 4 week interval. Overall, $10 \%$ and $40 \%$ of the patients, respectively, did best on a higher and lower dose than $2 \mathrm{~g} / \mathrm{kg} /$ month.

All patients started on monthly dosing but $30 \%$ changed to a different dosing interval, with $17 \%, 6 \%$, and $6 \%$ receiving the treatment at 3,6 , and 2 week intervals, respectively. In several cases, extending the treatment interval resulted in behavioral regression, requiring shortening the interval to restore treatment effectiveness (See Case \#3 below).

\section{Parent-reported outcomes}

The majority of the parents reported improvements in communication and/or language with fewer reporting improvements in aberrant behavior (Table 2). About a quarter reported improvement in repetitive behavior and academics. Between $10 \%$ and $30 \%$ reported improvements in social interactions, tics, motor function, and seizures, only three parents did not report improvements. Seventyone percent (22/31) of patients reported improvements in two or more symptoms and $90 \%$ (28/31) of the patients demonstrated improvements in one or more symptoms (Table 3).
Table 2 Parental Reported Symptomatic Improvements with Intravenous Immunoglobulin (IVIG) Treatment

\begin{tabular}{ll} 
Symptoms improvement & Patients reporting improvement \\
\hline Communication \& language & $58 \%(18 / 31)$ \\
Aberrant behavior & $35 \%(11 / 31)$ \\
Repetitive behavior & $23 \%(7 / 31)$ \\
Academics & $23 \%(7 / 31)$ \\
Social interactions & $23 \%(7 / 31)$ \\
Tics & $16 \%(5 / 31)$ \\
Motor & $16 \%(5 / 31)$ \\
Other & $16 \%(5 / 31)$ \\
Seizures & $10 \%(3 / 31)$ \\
None & $10 \%(3 / 31)$ \\
\hline
\end{tabular}

\section{Behavioral questionnaire outcomes}

For 21 and 20 of the treated children the SRS and ABC behavioral questionnaires were acquired both before and during IVIG treatment, respectively.

In Fig. 2a, all subscales of the SRS demonstrated improvement with IVIG treatment, although only a few of the improvements reached statistical significance (Fig. 2a). SRS subscales for cognition $\left(\mathrm{F}(1,57)=4.71, p<0.05, d^{\prime}=\right.$ $0.58)$ and mannerisms $\left(\mathrm{F}(1,57)=13.34, p<0.001, d^{\prime}=\right.$ 0.97 ) significantly improved with medium and large effect sizes, respectively. The total SRS score improved 
significantly with a medium effect size $(\mathrm{F}(1,57)=4.40, p$ $\left.=0.04, d^{\prime}=0.57\right)$. Change in communication $(\mathrm{F}(1,57)=$ $\left.2.60, p=0.11, d^{\prime}=0.43\right)$ and motivation $(\mathrm{F}(1,57)=2.99$, $\left.p=0.09, d^{\prime}=0.46\right)$ SRS subscales were borderline sig-

Table 3 Number of Symptoms Reported Improved with Intravenous Immunoglobulin (IVIG) Treatment

\begin{tabular}{ll}
\hline Number of patients & Number of symptoms improved \\
\hline $10 \%(3 / 31)$ & 0 \\
$19 \%(6 / 31)$ & 1 \\
$35 \%(11 / 31)$ & 2 \\
$13 \%(4 / 31)$ & 3 \\
$13 \%(4 / 31)$ & 4 \\
$6 \%(2 / 31)$ & 5 \\
$3 \%(1 / 31)$ & 6 \\
\hline
\end{tabular}

nificant with medium effect sizes, whereas the change in the awareness subscale was not statistically significant $(\mathrm{F}$ $\left.(1,57)=0.02, p=0.89, d^{\prime}=0.04\right)$.

In Fig. $2 b$, all subscales of the $A B C$ demonstrated improvement with IVIG treatment with all but one reaching statistical significance (Fig. 2b). ABC subscales for irritability $\left(\mathrm{F}(1,57)=5.81, p=0.02, d^{\prime}=0.64\right)$, lethargy (social withdrawal) $\left(\mathrm{F}(1,57)=5.75, p=0.02, d^{\prime}=0.64\right)$, hyperactivity $\left(\mathrm{F}(1,57)=4.63, \mathrm{p}=0.04, d^{\prime}=0.57\right)$ and inappropriate speech $\left(\mathrm{F}(1,57)=5.13, p=0.02, d^{\prime}=0.60\right)$ significantly improved with medium effect sizes. The total $\mathrm{ABC}$ score improved significantly with a large effect size $\left(\mathrm{F}(1,57)=9.88, p=0.003, d^{\prime}=0.83\right)$. The improvement in the $\mathrm{ABC}$ stereotypy subscales was not significant and had a small effect size $\left(\mathrm{F}(1,57)=1.90, p=0.17, d^{\prime}=0.36\right)$.

Relationship of treatment response to immune biomarkers

Biomarkers and behavioral questionnaires were available for 17 children for the SRS and 16 children for the

(A) Social Responsiveness Scale (SRS; $\mathrm{n}=21$ )

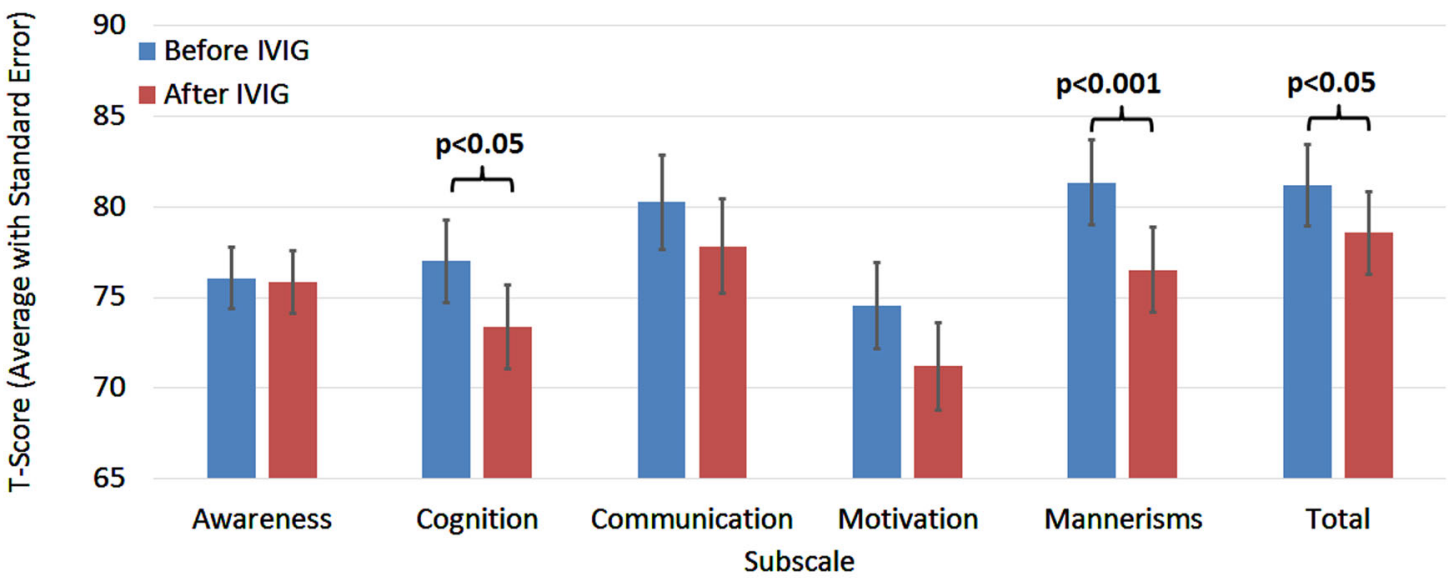

(B)
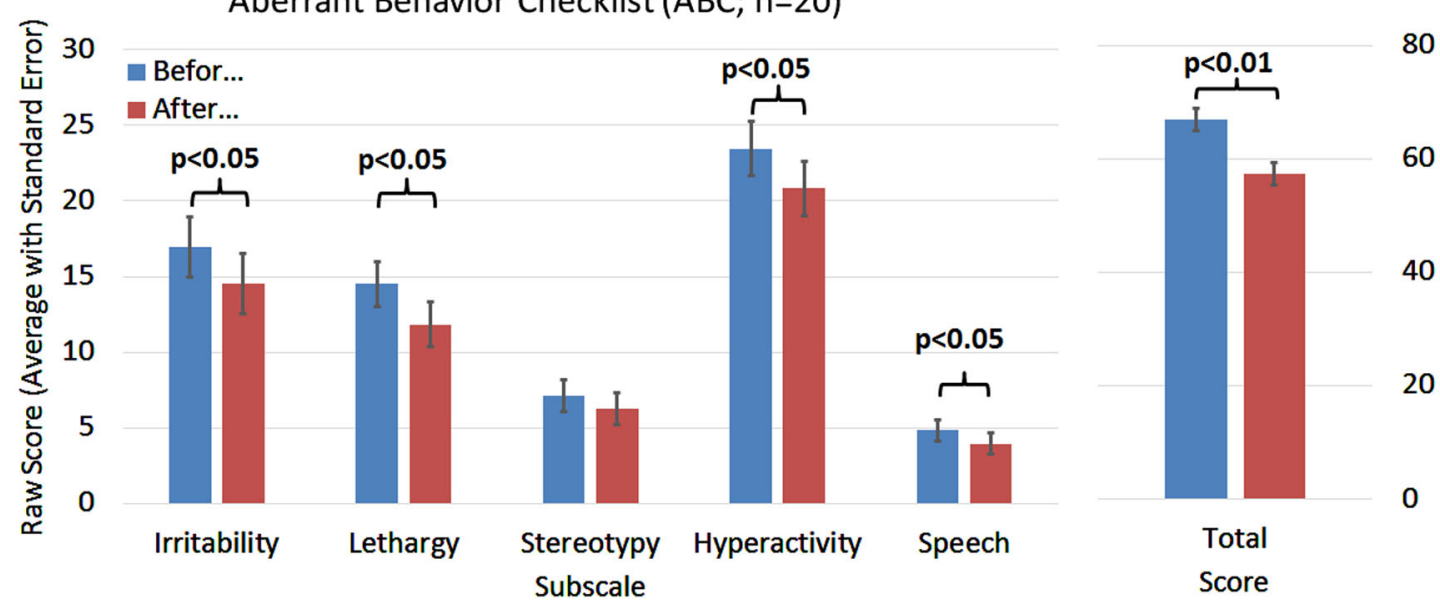

Fig. 2 Changes in the Social Responsiveness Scale (SRS) and Aberrant Behavior Checklist (ABC) with intravenous immunoglobulin (IVIG) treatment 

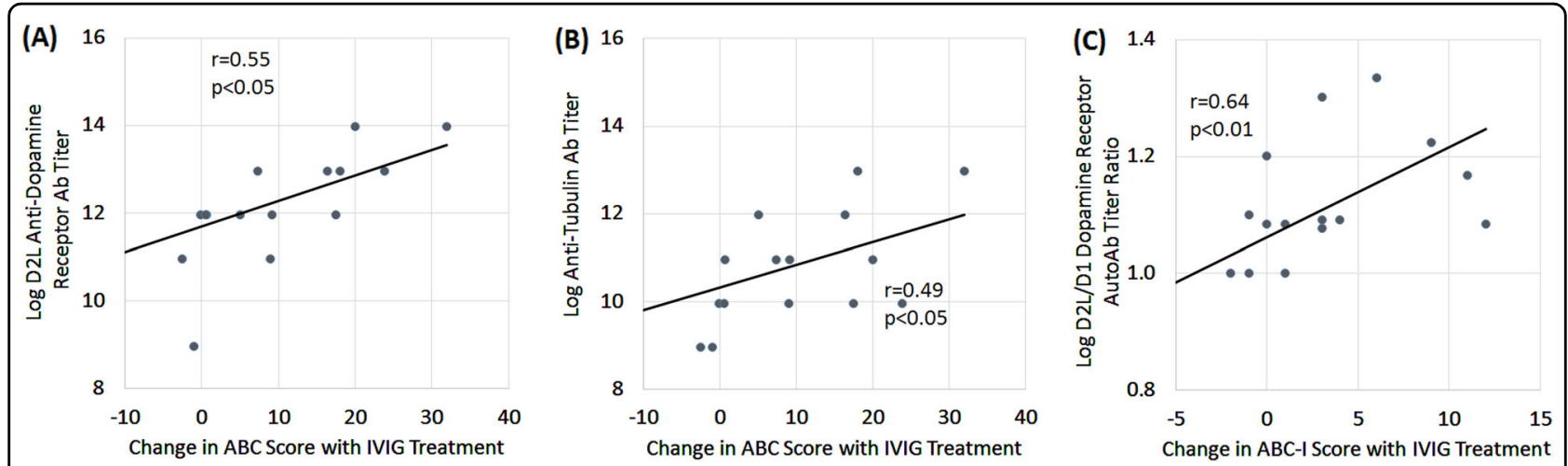

Fig. 3 Baseline antibody titers are related to improvement in Aberrant Behavior Checklist (ABC) scores following intravenous immunoglobulin (IVIG). Greater improvement in ABC was related to high titers of antidopamine D2 receptor (D2L) antidopamine and anti-tubulin autoantibodies. A higher ratio of D2L to D1 antidopamine antibody titer was related to greater improvements in the irritability subscale of the ABC with IVIG treatment

$\mathrm{ABC}$. Of the five biomarkers, the change in the total $\mathrm{ABC}$ score resulting from IVIG treatment was associated with the change in the anti-D2R antibody $(r=0.55, p<0.05$; Fig. 3a) and anti-tublin Antibody $(r=0.49, p<0.05$; Fig. 3b). In addition, as hypothesized, the anti-D2L titer/anti-D1 titer ratio was significantly associated with the Irritability subscale of the $\mathrm{ABC}(r=0.64, p<0.01$; Fig. 3c).

\section{IVIG responder analysis}

The change in SRS scores is modest when it occurs, whereas the change in $\mathrm{ABC}$ scores can be rather marked when it occurs (Fig. 4). Change in scores of the two questionnaires correlated well with each other $(r=0.52, p$ $=0.01$ ). Based on the distribution of scores a cutoff $\geq+1$ used to define a responder for the questionnaire scores. Using this criterion, five (25\%) individuals were IVIG nonresponders based on both questionnaires, three (15\%) were IVIG responders based on the SRS only, four (20\%) were IVIG responders based on the ABC only, and eight (40\%) were responders based on both questionnaires. A criterion of two or more reported improvements in symptoms was used as a cutoff for parental reported improvement. Using these criteria, $52 \%, 60 \%$, and $65 \%$ of patients were responders as defined by the SRS, $\mathrm{ABC}$, and parental report. Thus, we believe that the criterion we used separated the groups well into responders and nonresponders.

The Cunningham panel predicted response to IVIG treatment with an accuracy of $81 \%$ with a sensitivity of $90 \%$ and a specificity of $67 \%$ based on the ABC scores; with an accuracy of $88 \%$ with a sensitivity of $100 \%$ and a specificity of $75 \%$ based on the SRS scores; and with an accuracy of $88 \%$ with a sensitivity of $100 \%$ and a specificity of $67 \%$ based on parental scores.

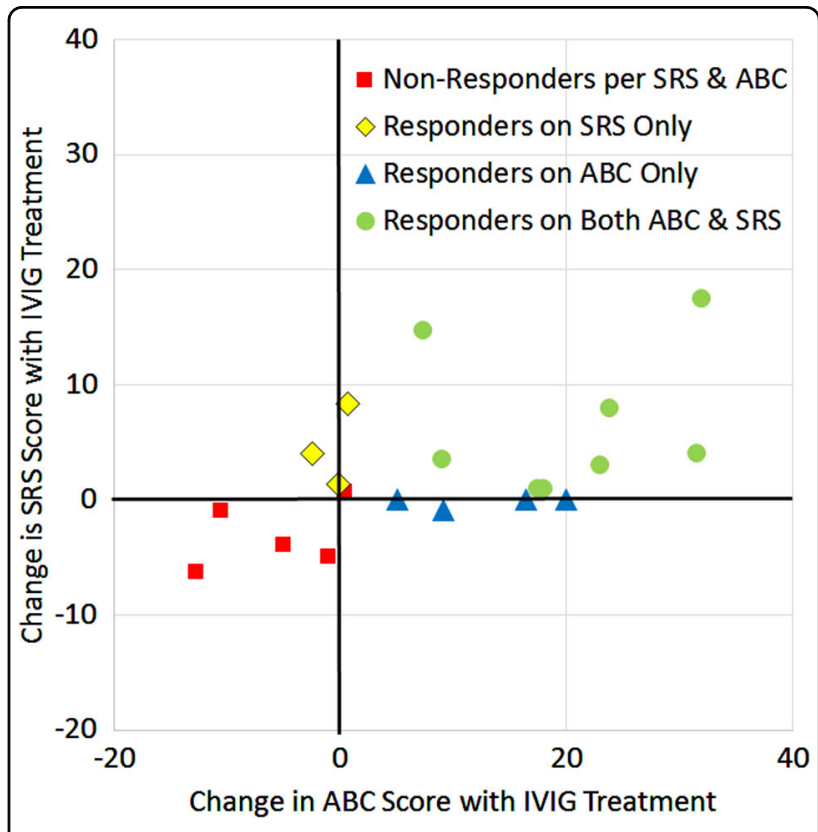

Fig. 4 Responders to intravenous immunoglobulin (IVIG) was defined by the Social Responsiveness Scale (SRS) and Aberrant Behavior Checklist (ABC). Red squares are non-responders based on either questionnaire. Yellow diamonds were responders only based on the SRS but not the ABC. Blue triangles were responders based on the $A B C$ but not the SRS. Green circles are responders based on both the $A B C$ and SRS

\section{Temporal characteristic of response}

The speed at which behavioral effects of the treatment arose and the duration of these effects were rather variable from patient to patient. However, $A B C$ responders (Fig. 5a) demonstrated a clear response that was variable in the rate at which it improved across patients, with some patients showing a very rapid decrease in $\mathrm{ABC}$ score and 

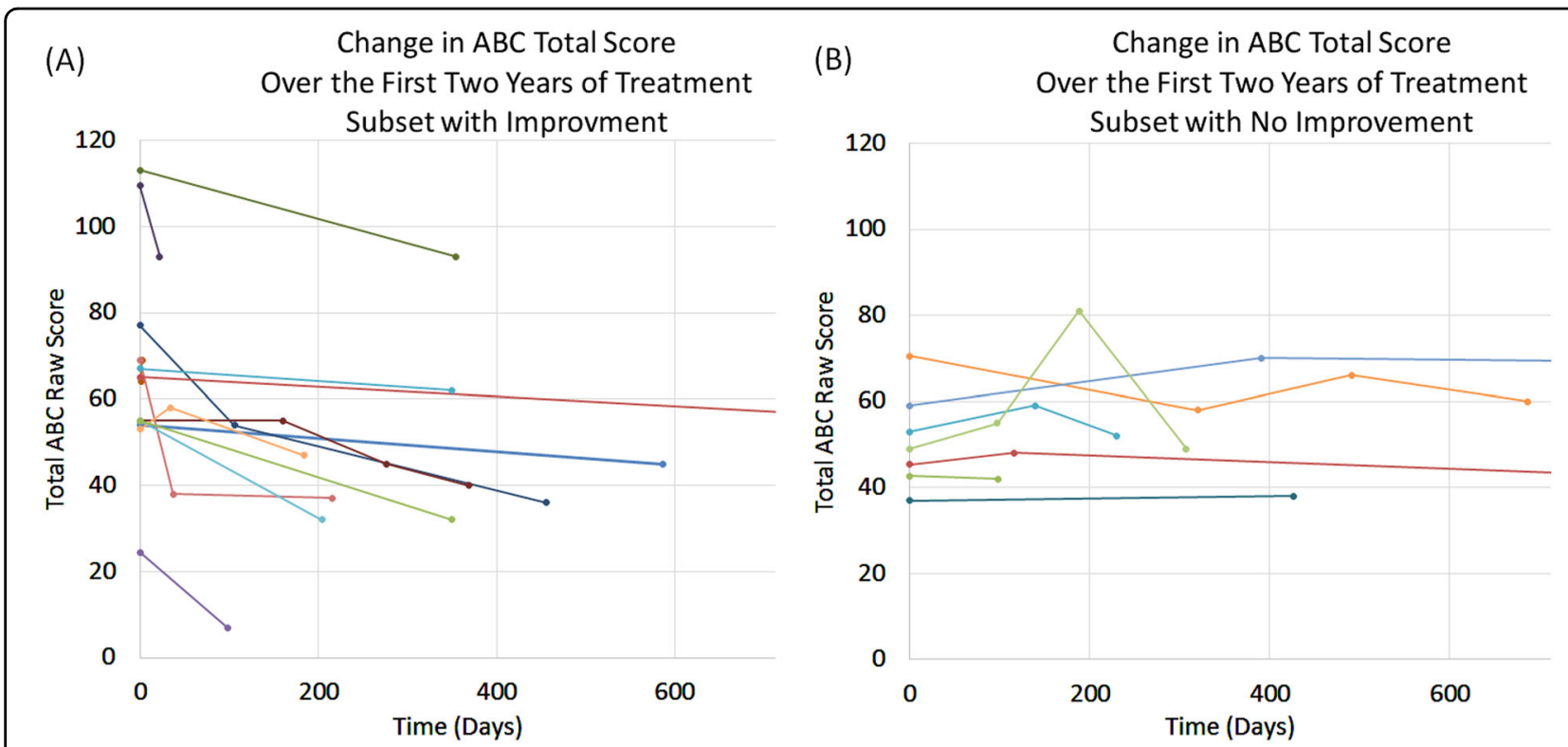

Fig. 5 Change in the total Aberrant Behavior Checklist (ABC) score over the first two years of treatment as compared to the average total $A B C$ score prior to treatment. The figure is divided up into (a) treatment responders and (b) treatment non-responders, as defined by the ABC

others showing a gradual improvement in $\mathrm{ABC}$ score. In contrast, non-responders showed a variable response with $\mathrm{ABC}$ score worsening and improving over time for many. One non-responder demonstrated a bout of severe hyperactivity that transiently increase the total $\mathrm{ABC}$ score dramatically.

\section{Long-term outcomes}

Of the 31 patients who were initially treated with IVIG, $77 \%(24 / 31)$ continued with IVIG treatment long-term (> 1 year). The majority of these 24 patients, $88 \%(21 / 24)$, found continuing benefit from the treatment. Of the seven that stopped the IVIG treatment, 29\% (2/7) stopped because of lack of insurance coverage, 29\% (2/7) stopped because adverse effects, $14 \%(1 / 7)$ stopped in order to try a more intense immunosuppression treatment, 14\% (1/7) stopped as a trial and requested to restart because of behavioral regression and $14 \%(1 / 7)$ was only prescribed one treatment. Only $6 \%(2 / 31)$ of the patients stopped the treatment because of lack of benefit and only $10 \%(3 / 13)$ who did not stop the treatment found limited continuing benefit.

\section{Adverse effects}

Sixty-five percent (20/31) of patients reported adverse effects, most commonly headaches and vomiting (Table 4). Many adverse effects were limited to the $48-72 \mathrm{~h}$ period immediately following the infusion. Treatments were individualized to each patient to minimize adverse effects by prescribing pre-infusion anti-pyretic (ibuprofen $10 \mathrm{mg} / \mathrm{kg}$ oral; PO), steroids (solumedrol $1 \mathrm{mg} / \mathrm{kg}$
Table 4 Adverse Effects Associated with Intravenous Immunoglobulin (IVIG) Treatment

\begin{tabular}{ll}
\hline Adverse effect & Patients reporting \\
\hline Headache & $39 \%(12 / 31)$ \\
Vomiting & $29 \%(9 / 31)$ \\
Worsening behavior & $16 \%(5 / 31)$ \\
Anxiety & $13 \%(4 / 31)$ \\
Fever & $13 \%(4 / 31)$ \\
Nausea & $10 \%(3 / 31)$ \\
Fatigue & $10 \%(3 / 31)$ \\
Rash & $6 \%(2 / 31)$ \\
\hline
\end{tabular}

intravaneous; IV), fluids ( $20 \mathrm{ml} / \mathrm{kg} 1 \frac{1}{2}$ normal saline over $1 \mathrm{~h}$ ), anti-emetics (ondansetron $0.15 \mathrm{mg} / \mathrm{kg} \mathrm{IV}$ ), antihistamine (diphenhydramine $0.5 \mathrm{mg} / \mathrm{kg} \mathrm{IV} / \mathrm{PO}$ ) and/or post infusion fluids. These pretreatments were prescribed in $84 \%, 81 \%, 32 \%, 26 \%, 19 \%$, and $6 \%$, respectively. Three patients were prescribed anti-emetics up to $72 \mathrm{~h}$ post infusion. One patient with baseline idiopathic hypertension was given aggressive hydration pre and post infusion to prevent fluctuation in blood pressure.

\section{Selected example cases}

Case 1

At 15 months of age, a male patient experienced sudden regression in speech, eye contact, and fine motor skills. After 6 years of speech and occupational therapy, he could speak 3-4 word sentences but was not able to engage in 


\section{Case \#1 - Mitochondrial Dysfunction and Autoimmune Encephalopathy}

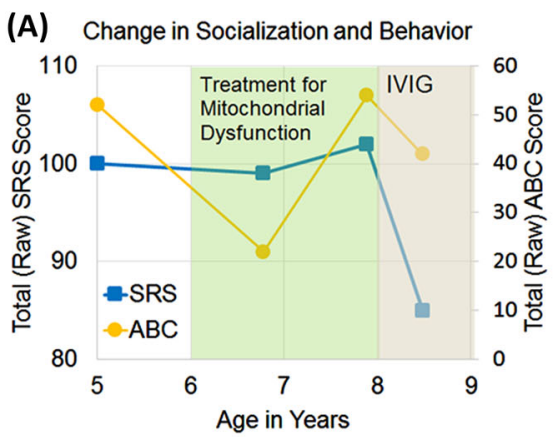

(B) After 5 years of Occupational Therapy

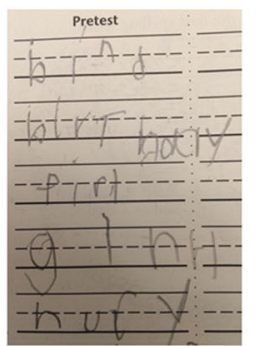

After 2 days of IVIG Treatment

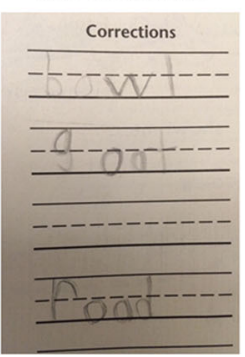

Case \#2 - N-Type Calcium Channel Antibody

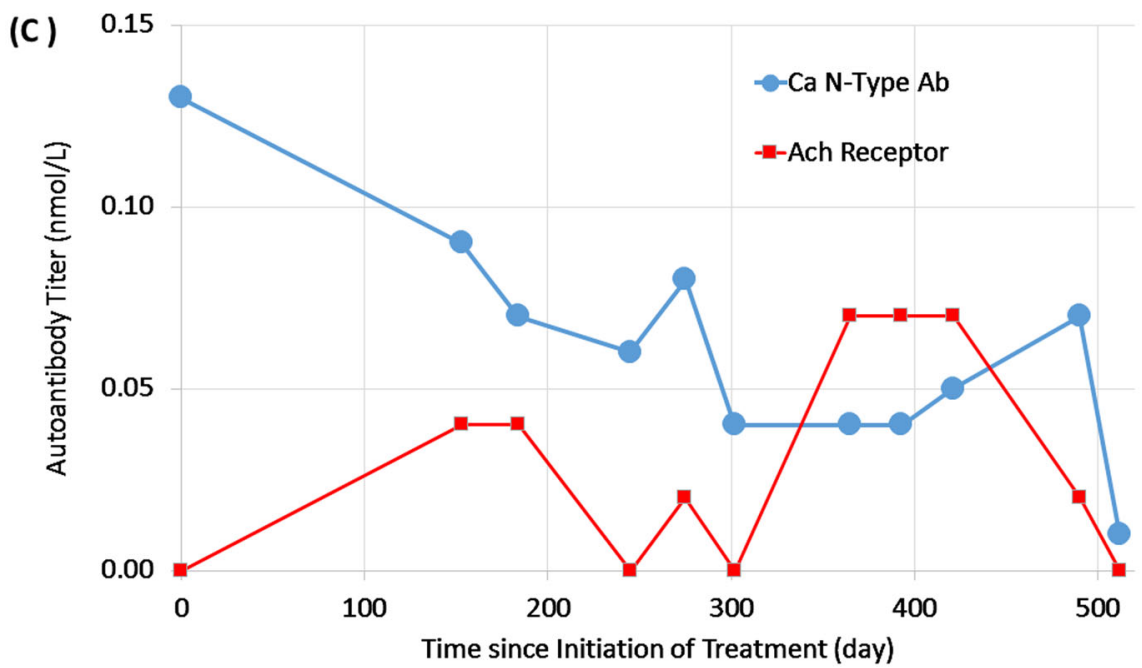

Case \#3 - Behavioral Relapse with Change in Treatment Interval

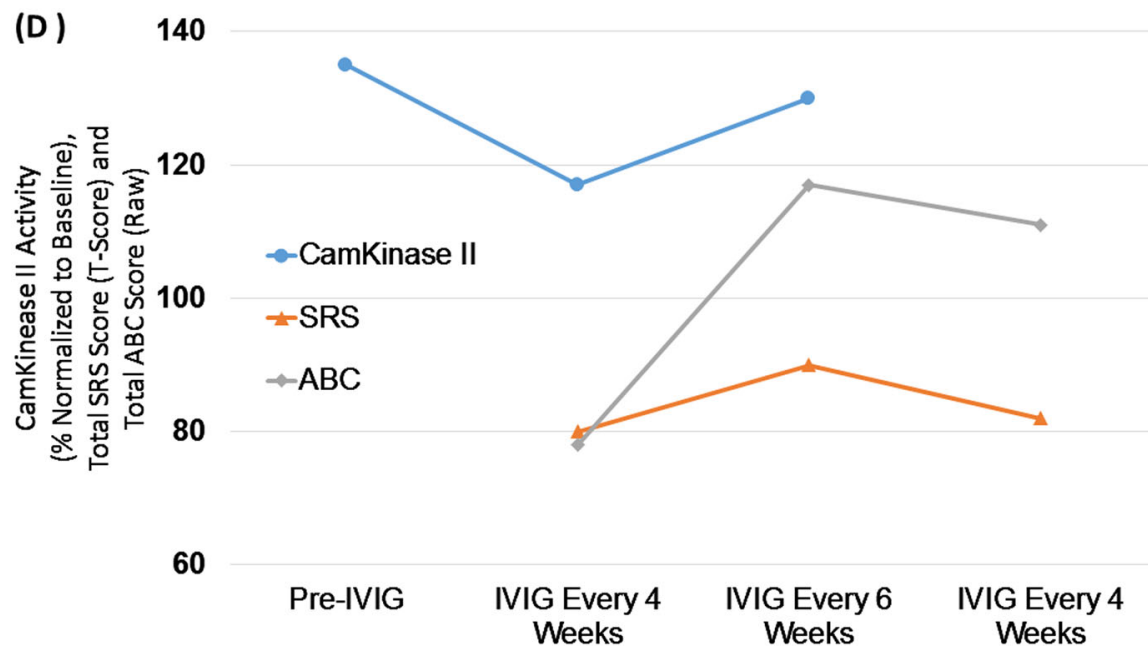

Fig. 6 Case examples of responders to intravenous immunoglobulin (IVIG) treatment. Case \#1 is a child with autism spectrum disorder (ASD) and mitochondrial dysfunction that had a sudden worsening in behavior at $\mathbf{8}$ years of age. a Initiating IVIG significantly improved behavior as measured by the Aberrant Behavior Checklist (ABC) and social function as measured by the Social Responsiveness Scale (SRS). b The writing sample on the left shows the patient's handwriting a few months before IVIG treatment, and the sample on the right shows the patient's handwriting 2 days after his first IVIG treatment. Case \#2 is a boy with sudden regression found to have an N-Type Voltage-Gated Calcium-Channel autoantibody. c IVIG treatment significantly improved autoantibody titers. Case \#3 is a girl with multiple regressions and severe ASD and related symptoms. $\mathbf{d}$ IVIG normalized CamKinase II activity but CamKinase II activity increased to abnormal levels and behavior, as measured by the ABC and SRS, worsened with increasing the IVIG treatment interval to every 6 weeks. Behavior improved after reinstating the original every 4 weeks dosing 
reciprocal conversation, exhibited limited eye contact, and suffered from severe restricted interests, anxiety, and frequent meltdowns. A medical evaluation demonstrated that he had biomarkers consistent with mitochondrial dysfunction, as manifested by repeated elevated lactate, pyruvate, and alanine-to-lysine ratio (mean $(N, \mathrm{SD})$ $2.3 \mathrm{mM} / \mathrm{L}(25,0.65), \mathrm{nl}<1.3 ; 0.20 \mathrm{mM} / \mathrm{L}(24,0.5), \mathrm{nl}<0.08$; $4.0(26,1.96), \mathrm{nl}<2.5$, respectively) and mitochondrial buccal swab demonstrating depressed citrate synthase (55\%) and elevated complex I (178\%) and IV (236\%) function. A mitochondrial cocktail was started, including ubiquinol, L-carnitine, creatine monophosphate, niacin, pantothenic acid, pyridoxine, riboflavin, thiamine, cobalamin, and folinic acid. During the first treatment year he demonstrated improved adaptability, socialization, and ASD-related behavior. However, improvements in socialization plateaued and behavior worsened (See Fig. 6a), prompting further investigation. A Cunningham panel showed elevations in anti-tubulin antibodies (2000; $\mathrm{nl}=$ 250-1000) and CaMKII (151, $\mathrm{nl}<130$ ), prompting a trial of IVIG at $1 \mathrm{~g} / \mathrm{kg} /$ day $\times 2$ days every month. Within 2 days of the IVIG treatment, his handwriting significantly improved (See Fig. 6b). With each subsequent treatment he progressively gained skills, including significant improvements in language. After 5 months, he began having reciprocal conversations and was able to describe how IVIG made him "feel". After 6 months, his restricted interests resolved. After 18 months of IVIG treatment, he has been able to sit with peers in a noisy gymnasium and engage in social conversation as part of a small group.

\section{Case 2}

At $\sim 3^{1 / 2}$ years of age, a previously normally developing child underwent a slow regression lasting 6 months. His parents pursued a language assessment at the beginning of the regression because he became less verbal but the evaluation did not demonstrate any significant abnormalities. However, as the third year of his life progressed, he lost speech, social interactions, developed repetitive movements, and sleep disturbance with an inability to stay asleep throughout the night. A repeat language evaluation at 3 years 11 months demonstrated a significant (17 standardized point) loss in language ability as compared with the evaluation earlier in the year. At 4 years of age he was diagnosed with ASD. Gluten- and dairy-free diet reportedly improved focus and attention and speech, occupational, physical, and applied behavioral analysis therapies began. Family history was remarkable for learning disabilities in maternal family and developmental delays in the paternal family, but the mother, father, and sibling did not have a history of neurodevelopmental disorders. He was otherwise healthy.
He was evaluated in our clinic at 4 years 6 months of age. On examination, he was active, cooperative, and anxious with very limited verbal communication skills but demonstrated eye contact. A mitochondrial and genetic workup (Chromosomal Microarray and Fragile X) and overnight EEG were unremarkable. FRA, NMDA, and GAD65 antibodies were negative, however, PNP demonstrated an elevated N-Type Calcium-Channel antibody $(0.13 \mathrm{nmol} / \mathrm{L} ; \mathrm{nl}<0.03 \mathrm{nmol} / \mathrm{L})$. A magnetic resonance imaging scan of the brain was normal and lumbar puncture demonstrated low protein $(16 ; \mathrm{nl} \geq 20$ ).

IVIG treatment was initiated at $1 \mathrm{~g} / \mathrm{kg} /$ day for 2 days. Four weeks after the first IVIG infusion a marked improvement occurred in near constant chewing, repetitive movement, and severe sleep disruption. Five months after the first treatment, some ASD symptoms started to return, resulting in starting IVIG treatments on a monthly to every other month schedule. The N-type VGCC antibody dropped to $0.09 \mathrm{nmol} / \mathrm{l}$ just prior to the second IVIG treatment and then progressively further with additional treatments, reaching a low of $0.4 \mathrm{nmol} / \mathrm{l}$ for several months, and eventually dropping to $0.01 \mathrm{nmol} / \mathrm{L}$ (Fig. 6c). The ABC total score dropped from 69 before treatment to 38 just before the second treatment with this decrease paralleling the decrease in the N-type VGCC antibody. Interestingly, there was an increase in the neuronal acetylcholine receptor autoantibody during the treatments course, which eventually decreased along with the N-type VGCC antibody.

\section{Case 3}

This girl developed normally until about 3 years of age when she experienced periodic episodes of abrupt loss of previously acquired skills and developed other neuropsychiatric symptoms. The most significant of these regressions was at 4 years of age when she developed an abrupt onset of repetitive behaviors, tics, poor concentration, emotional liability, separation anxiety, urinary frequency and urgency, sleep disturbance, and aggressive behavior. She was diagnosed with ASD at age 8 years of age. At 9 years of age she developed partial complex seizures, incompletely controlled by lamotrigine. At 7 years of age, a Cunningham panel demonstrated a CaMKII $(135 ; \mathrm{nl}<130$; See Fig. 6d) elevation. She was treated with IVIG $1 \mathrm{~g} / \mathrm{kg} /$ day for 2 days every month. Improvements occurred in tics, academics, and seizure frequency, and CaMKII normalized (Fig. 6d). After 6 months of treatment, the interval of IVIG was extend to every 6 weeks. The child's behavior quickly regressed with a significant increase in both the SRS and ABC scales and an increase in the CaMKII in the abnormal range again (Fig. 6d). After 6 months on monthly IVIG, her behavior improved (Fig. 6d). 


\section{Discussion}

In this study, we examined 82 children with neurodevelopmental disorders for biomarkers consistent with AIE and considered for immunomodulatory treatment for those with a clinical presentation and laboratory values consistent with AIE. Of those who underwent IVIG treatment using our care team, the majority had symptoms improvements as measured by standardized questionnaires. Although adverse effects were reported for the majority of patients, they were mostly limited to the time around the IVIG infusion, resulting in the great majority of patients considering the benefits outweighing any adverse effects.

Interestingly, in our cohort of patients who presented to our ASD clinic, very few demonstrated autoantibodies usually associated with AIE in children. Interestingly, none of the patients was positive for NMDA receptor or VGKC autoantibodies. Only $5 \%$ of the patient cohort was positive for the GAD65 autoantibody with only one GAD65 titer within a clinically significant range. This low rate of positivity could explain the contradictory reports of the association of the GAD65 autoantibody with ASD as the rate of positivity may be very dependent on the sample of ASD children studied ${ }^{40,60}$. The child with ASD and the highest GAD65 titer also had type 1 diabetes, which is a disorder known to be associated with the GAD65 autoantibody ${ }^{31}$. This child was a responder to IVIG. Interestingly, GAD65 may be functionally disrupted in ASD in other ways besides an autoantibody mechanism. For example, GAD65 is downregulated in the prenatal valproic acid and MIA animal models of ASD ${ }^{61-65}$ and in post-mortem brain from individuals with $\mathrm{ASD}^{66,67}$, suggesting that it is a potentially important target of pathology in individuals with ASD.

This is the first reported association of VGCC autoantibodies with ASD. The VGCC is associated with a wide range of cognitive and motor disturbances ${ }^{68}$ and cerebellar degeneration in childhood ${ }^{69}$. The child positive for the N-type VGCC demonstrated an abrupt onset of sleep disturbance, repetitive behavior, and chewing on objects in addition to language and social regression at the onset of this disorder. IVIG treatment significantly and quickly improved these symptoms. Interestingly, like the GAD enzyme, ASD has been associated with non-autoimmune disruption in calcium-channel function ${ }^{70}$. Thus, some autoantibodies in individuals with ASD may merely represent one mechanism that can disrupt common pathophysiological disturbances leading to ASD.

Overall, the majority of parents of the patients treated with IVIG found the treatment beneficial and believed that the benefits outweighed any adverse effects. Close observation was important to tailor the dose and frequency of the treatment and minimize adverse effects. Consistent with previous literature, IVIG treatment in this study improved scores on the $\mathrm{ABC}^{41,44}$ and social interactions ${ }^{42}$. Of the six studies using IVIG in the treatment of ASD, three open-label studies, totaling 63 patients ${ }^{41-43}$ and one double-blind placebo-controlled study totaling 12 patients ${ }^{44}$ demonstrated a benefit, whereas two smaller open-label studies, totaling 15 patients ${ }^{45,46}$, did not show benefit. Two of the studies that demonstrated benefit selected individuals with immune system abnormalities, like the current study ${ }^{42,43}$. In contrast, the two open-label studies that did not demonstrate benefit did not select for immune abnormalities ${ }^{45,46}$. In addition, all previous studies used a lower dose of IVIG, usually $0.4 \mathrm{~g} / \mathrm{kg}$ monthly. Some have observed better response with higher doses of IVIG $^{71}$ potentially explaining the high rate of response in our study. Clearly more research is needed to better understand which subset of children with ASD can best benefit from IVIG as well as the optimal dose and interval for the treatment.

The majority of the ASD patients who had autoantibodies demonstrated elevations in autoantibodies measured by the Cunningham panel along with an elevation in the activation of CaMKII. Our analysis suggests that two of the autoantibodies, the anti-tubulin and antiD2R were associated with responsiveness to IVIG treatment, suggesting that these could be biomarkers to select individuals who might benefit most from IVIG treatment. It should be noted that the participants were selected based on an elevated CaMKII, so this biomarker may have been artificially fixed with a limited dynamic range because of our patient selection process, thereby decreasing its ability to be a predictive biomarker. As the $\mathrm{ABC}$ was most closely associated with immune biomarkers, it may be that the autoantibodies identified as predictive of response to IVIG may be closely associated with modulation of behavior as has been suggested in Sydenham chorea $^{22}$ and PANDAS ${ }^{23,26}$.

The limitations of this study include the small sample size and the questionnaire-based outcome measures. Furthermore, this study was not a systematic clinical trial with comprehensive measurements of behavior, cognition, and language. Thus, further studies are needed to better identify which specific symptoms are targeted by IVIG treatment as well as the most-sensitive biomarkers to identify which patients are most likely to respond to IVIG treatment. Despite these limitations, our study verifies previous reports that suggest that IVIG may be useful in individuals with ASD who demonstrate biomarkers of immune abnormalities.

\section{Acknowledgements}

This research was supported, in part, by the Autism Research Institute (San Diego, CA), the Arkansas Biosciences Institute (Little Rock, AR), the Jonty Foundation (St Paul, MN), the Gupta Family Foundation (Atherton, CA), the Jager Family Foundation (Chicago, IL), the Jane Botsford Johnson Foundation (New York, NY), and the Arkansas Children's Research Institute (Little Rock, AR). 
None of the sponsors were involved with the design or conduct of the study, collection, management, analysis, or interpretation of the data; or preparation, review, approval of the manuscript or decision to submit the manuscript for publication. The authors had full access to all of the data in the study and take responsibility for the integrity of the data and the accuracy of the data analysis.

\section{Author details}

'University of Arkansas for Medical Sciences, Little Rock, AR 77205, USA ${ }^{2}$ BioROSA Technologies Inc, San Francisco, CA 94103, USA. ${ }^{3}$ Department of Biomedical Engineering, Rensselaer Polytechnic Institute, Troy, NY 12180, USA. ${ }^{4}$ Center for Biotechnology \& Interdisciplinary Studies, Rensselaer Polytechnic Institute, Troy, NY 12180, USA. ${ }^{5}$ Department of Microbiology and Immunology, University of Oklahoma Health Sciences Center, Oklahoma City, OK 73104, USA ${ }^{6}$ Moleculera Labs, Inc, Oklahoma City, OK, USA. ${ }^{7}$ Barrow Neurological Institute at Phoenix Children's Hospital, Phoenix, AZ, USA. ${ }^{8}$ Department of Child Health, University of Arizona College of Medicine - Phoenix, Phoenix, AZ, USA

\section{Author contributions}

Kathleen Connery, Leanna Delhey, Shannon Rose, John Slattery, Stephen Kahler, and Richard E. Frye designed the study and wrote the protocol, collected the data, analyzed the data, drafted the manuscript. Madeleine W. Cunningham and Craig Shimasaki contributed to interpreting the data and writing the manuscript. All authors contributed to and have approved the final manuscript.

\section{Conflict of interest}

Kathleen Connery, Leanna Delhey, John autoantibody have no conflicts of interest to declare. Craig Shimasaki is the President \& Chief Executive Officer and Madeleine W. Cunningham is the Chief Scientific Officer of Moleculera Labs, Inc., which provides the Cunningham Panel ${ }^{\mathrm{TM}}$, an immune panel that is used in this study. Richard E Frye is on the Scientific Advisory Board of lliad Neurosciences, Inc, which performs the folate receptor alpha autoantibody which was used in some patients in this study.

\section{Publisher's note}

Springer Nature remains neutral with regard to jurisdictional claims in published maps and institutional affiliations.

Supplementary Information accompanies this paper at (https://doi.org/ 10.1038/s41398-018-0214-7).

Received: 22 March 2018 Revised: 7 June 2018 Accepted: 20 June 2018 Published online: 10 August 2018

\section{References}

1. Zablotsky, B., Black, L. I., Maenner, M. J., Schieve, L. A., \& Blumberg, S. J. Estimated prevalence of autism and other developmental disabilities following questionnaire changes in the 2014 National Health Interview Survey. National Health Stat. Reports 13, 1-20 (2015).

2. Reichow, B., Barton, E. E., Boyd, B. A. \& Hume, K. Early intensive behavioral intervention (EIBI) for young children with autism spectrum disorders (ASD). Cochrane Database Syst. Rev. 10, CD009260 (2012).

3. Rossignol, D. A. \& Frye, R. E. A review of research trends in physiological abnormalities in autism spectrum disorders: immune dysregulation, inflammation, oxidative stress, mitochondrial dysfunction and environmental toxicant exposures. Mol. Psychiatry 17, 389-401 (2012).

4. Foley, K. A., MacFabe, D. F., Kavaliers, M. \& Ossenkopp, K. P. Sexually dimorphic effects of prenatal exposure to lipopolysaccharide, and prenatal and postnatal exposure to propionic acid, on acoustic startle response and prepulse inhibition in adolescent rats: relevance to autism spectrum disorders. Behav. Brain Res. 278, 244-256 (2015).

5. Foley, K. A., Ossenkopp, K. P., Kavaliers, M. \& Macfabe, D. F. Pre- and neonatal exposure to lipopolysaccharide or the enteric metabolite, propionic acid, alters development and behavior in adolescent rats in a sexually dimorphic manner. PLOS. ONE 9, e87072 (2014).

6. Derecki, N. C. et al. Wild-type microglia arrest pathology in a mouse model of Rett syndrome. Nature 484, 105-109 (2012).
7. Masi, A., Quintana, D. S., Glozier, N., Lloyd, A. R., Hickie, I. B. \& Guastella, A. J. Cytokine abberations in autism spectrum disorder: a systematic review and meta-analysis. Mol. Psychiatry 20, 440-446 (2015).

8. Rossignol, D. A. \& Frye, R. E. Evidence linking oxidative stress, mitochondrial dysfunction, and inflammation in the brain of individuals with autism. Front. Physiol. 5, 150 (2014).

9. Frye R. E., Slattery J. C., Quadros E. V. Folate metabolism abnormalities in autism: potential biomarkers. Biomark Med. (2017). https://doi.org/10.2217/ bmm-2017-0109.

10. Bashir, S. \& Al-Ayadhi, L. Endothelial antibody levels in the sera of children with autism spectrum disorders. J. Chin. Med. Assoc. 78, 414-417 (2015).

11. Careaga, M., Hansen, R. L., Hertz-Piccotto, I., Van de Water, J. \& Ashwood, P. Increased anti-phospholipid antibodies in autism spectrum disorders. Mediat. Inflamm. 2013, 935608 (2013).

12. Goines, P. et al. Autoantibodies to cerebellum in children with autism associate with behavior. Brain Behav. Immun. 25, 514-523 (2011).

13. Mostafa, G. A. \& Al-Ayadhi, L. Y. Increased serum levels of anti-ganglioside M1 auto-antibodies in autistic children: relation to the disease severity. J. Neuroinflamm. 8, 39 (2011).

14. Mostafa, G. A. \& Al-Ayadhi, L. Y. The relationship between the increased frequency of serum antineuronal antibodies and the severity of autism in children. Eur. J. Paediatr. Neurol. 16, 464-468 (2012).

15. Mostafa, G. A. \& Kitchener, N. Serum anti-nuclear antibodies as a marker of autoimmunity in Egyptian autistic children. Pediatr. Neurol. 40, 107-112 (2009).

16. Braunschweig, D. et al. Autism-specific maternal autoantibodies recognize critical proteins in developing brain. Transl. Psychiatry 3, e277 (2013).

17. Piras, I. S., Haapanen, L., Napolioni, V., Sacco, R., Van de Water, J. \& Persico, A. M. Anti-brain antibodies are associated with more severe cognitive and behavioral profiles in Italian children with Autism Spectrum Disorder. Brain Behav. Immun. 38, 91-99 (2014).

18. Nordahl, C. W. et al. Maternal autoantibodies are associated with abnormal brain enlargement in a subgroup of children with autism spectrum disorder. Brain Behav. Immun. 30, 61-65 (2013).

19. Greengard, P., Valtorta, F., Czernik, A. J. \& Benfenati, F. Synaptic vesicle phosphoproteins and regulation of synaptic function. Science 259, 780-785 (1993).

20. Griffith, L. C. \& Schulman, H. The multifunctional Ca2 +/calmodulin-dependent protein kinase mediates Ca2 +-dependent phosphorylation of tyrosine hydroxylase. J. Biol. Chem. 263, 9542-9549 (1988).

21. Chakrabarty, K., Bhattacharyya, S., Christopher, R. \& Khanna, S. Glutamatergic dysfunction in OCD. Neuropsychopharmacology 30, 1735-1740 (2005).

22. Kirvan, C. A., Swedo, S. E., Heuser, J. S. \& Cunningham, M. W. Mimicry and autoantibody-mediated neuronal cell signaling in Sydenham chorea. Nat. Med. 9, 914-920 (2003).

23. Kirvan, C. A., Swedo, S. E., Snider, L. A. \& Cunningham, M. W. Antibodymediated neuronal cell signaling in behavior and movement disorders. J. Neuroimmunol. 179, 173-179 (2006).

24. Kury, S. et al. De novo mutations in protein kinase genes CAMK2A and CAMK2B cause intellectual disability. Am. J. Hum. Genet. 101, 768-788 (2017).

25. Cox, C. J. et al. Antineuronal antibodies in a heterogeneous group of youth and young adults with tics and obsessive-compulsive disorder. J. Child Adolesc. Psychopharmacol. 25, 76-85 (2015).

26. Ben-Pazi, H., Stoner, J. A. \& Cunningham, M. W. Dopamine receptor autoantibodies correlate with symptoms in Sydenham's chorea. PLOS. ONE 8, e73516 (2013).

27. Akman, C. I., Patterson, M. C., Rubinstein, A. \& Herzog, R. Limbic encephalitis associated with anti-GAD antibody and common variable immune deficiency. Dev. Med. Child Neurol. 51, 563-567 (2009).

28. Chou, I. J. et al. Limbic encephalitis in Taiwanese children and adolescence: a single center study. Pediatr. Neonatol. 54, 246-253 (2013).

29. Haberlandt, E et al. Limbic encephalitis in children and adolescents. Arch. Dis. Child. 96, 186-191 (2011).

30. Lin, J. J. et al. Antiglutamic acid decarboxylase antibodies in children with encephalitis and status epilepticus. Pediatr. Neurol. 47, 252-258 (2012).

31. Olson, J. A., Olson, D. M., Sandborg, C., Alexander, S. \& Buckingham, B. Type 1 diabetes mellitus and epilepsia partialis continua in a 6-year-old boy with elevated anti-GAD65 antibodies. Pediatrics 109, E50 (2002).

32. Saltik, S., Turkes, M., Tuzun, E., Cakir, A. \& Ulusoy, C. Peripheral neuropathy associated with antiglutamic acid decarboxylase antibodies. Pediatr. Neurol. 48 403-406 (2013). 
33. Sanefuji, M. et al. A case of childhood stiff-person syndrome with striatal lesions: a possible entity distinct from the classical adult form. Brain Dev. $\mathbf{3 5}$ 575-578 (2013).

34. Hacohen, Y. et al. Paediatric autoimmune encephalopathies: clinical features, laboratory investigations and outcomes in patients with or without antibodies to known central nervous system autoantigens. J. Neurol. Neurosurg. Psychiatry 84, 748-755 (2013).

35. Titulaer, M. J. et al. Treatment and prognostic factors for long-term outcome in patients with anti-NMDA receptor encephalitis: an observational cohort study. Lancet Neurol. 12, 157-165 (2013).

36. Scott, O. et al. Anti-N-methyl-D-aspartate (NMDA) receptor encephalitis: an unusual cause of autistic regression in a toddler. J. Child Neurol. 29, 691-694 (2014).

37. Creten, C. et al. Late onset autism and anti-NMDA-receptor encephalitis. Lancet (Lond., Engl.). 378, 98 (2011).

38. Hacohen, Y. et al. N-methyl-d-aspartate (NMDA) receptor antibodies encephalitis mimicking an autistic regression. Dev. Med. Child Neurol. 58, 1092-1094 (2016).

39. Najjar, S. et al. Neuropsychiatric autoimmune encephalitis without VGKCcomplex, NMDAR, and GAD autoantibodies: case report and literature review. Cogn. Behav. Neurol. 26, 36-49 (2013).

40. Rout, U. K., Mungan, N. K. \& Dhossche, D. M. Presence of GAD65 autoantibodies in the serum of children with autism or ADHD. Eur. Child Adolesc. Psychiatry 21, 141-147 (2012).

41. Boris, M., Goldblatt, A. \& Edelson, S. M. Improvements in children with autism treated with intravenous gamma globulin. J. Nutr. Environ. Med. 15, 169-176 (2005).

42. Gupta, S., Aggarwal, S. \& Heads, C. Dysregulated immune system in children with autism: beneficial effects of intravenous immune globulin on autistic characteristics. J. Autism Dev. Disord. 26, 439-452 (1996).

43. Oleske J. Another view of autism. UNMDNJ Research: 22-23, Winter 2004.

44. Niederhofer, H., Staffen, W. \& Mair, A. Immunoglobulins as an alternative strategy of psychopharmacological treatment of children with autistic disorder. Neuropsychopharmacology 28, 1014-1015 (2003).

45. DelGiudice-Asch, G., Simon, L., Schmeidler, J., Cunningham-Rundles, C. \& Hollander, E. Brief report: a pilot open clinical trial of intravenous immunoglobulin in childhood autism. J. Autism Dev. Disord. 29, 157-160 (1999).

46. Plioplys, A. V. Intravenous immunoglobulin treatment of children with autism. J. Child Neurol. 13, 79-82 (1998).

47. Perlmutter, S. J. et al. Therapeutic plasma exchange and intravenous immunoglobulin for obsessive-compulsive disorder and tic disorders in childhood. Lancet (Lond., Engl.). 354, 1153-1158 (1999).

48. Perlmutter, S. J. et al. A case of pediatric autoimmune neuropsychiatric disorders associated with streptococcal infections. Am. J. Psychiatry 155 1592-1598 (1998).

49. Kovacevic, M., Grant, P. \& Swedo, S. E. Use of intravenous immunoglobulin in the treatment of twelve youths with pediatric autoimmune neuropsychiatric disorders associated with streptococcal infections. J. Child Adolesc. Psychopharmacol. 25, 65-69 (2015).

50. Delhey L. M., et al. The effect of mitochondrial supplements on mitochondrial activity in children with autism spectrum disorder. J. Clin. Med. 6, E18 (2017)

51. Frye, R. E. et al. Blocking and binding folate receptor alpha autoantibodies identify novel autism spectrum disorder subgroups. Front. Neurosci. 10, 80 (2016).

52. Frye, R. E. et al. Folinic acid improves verbal communication in children with autism and language impairment: a randomized double-blind placebo-controlled trial. Mol. Psychiatry 23, 247-256 (2016).
53. Frye R. E., et al. Thyroid dysfunction in children with autism spectrum disorder is associated with folate receptor alpha autoimmune disorder. J. Neuroendocrinol. 29 (2017).

54. Grea, $\mathrm{H}$. et al. Clinical and autoimmune features of a patient with autism spectrum disorder seropositive for anti-NMDA-receptor autoantibody. Dialogues Clin. Neurosci. 19, 65-70 (2017).

55. Frye, R. E., Sequeira, J. M., Quadros, E. V., James, S. J. \& Rossignol, D. A. Cerebral folate receptor autoantibodies in autism spectrum disorder. Mol. Psychiatry $\mathbf{1 8}$, 369-381 (2013).

56. Frye, R. E. et al. Metabolic effects of sapropterin treatment in autism spectrum disorder: a preliminary study. Transl. Psychiatry 3, e237 (2013).

57. Kaat, A. J., Lecavalier, L. \& Aman, M. G. Validity of the aberrant behavior checklist in children with autism spectrum disorder. J. Autism Dev. Disord. 44, 1103-1116 (2014)

58. Constantino, J. N. The Social Responsiveness Scale. Western Psychological Services, Los Angeles, 2002).

59. Murray, M. J., Mayes, S. D. \& Smith, L. A. Brief report: excellent agreement between two brief autism scales (Checklist for Autism Spectrum Disorder and Social Responsiveness Scale) completed independently by parents and the autism diagnostic interview-revised. J. Autism Dev. Disord. 41, 1586-1590 (2011).

60. Kalra, S. et al. No evidence of antibodies against GAD65 and other specific antigens in children with autism. BBA Clin. 4, 81-84 (2015).

61. Chau, D. K., Choi, A. Y., Yang, W., Leung, W. N. \& Chan, C. W. Downregulation of glutamatergic and GABAergic proteins in valproric acid associated social impairment during adolescence in mice. Behav. Brain Res. 316, 255-260 (2017).

62. Wei, R. et al. A single low dose of valproic acid in late prenatal life alters postnatal behavior and glutamic acid decarboxylase levels in the mouse. Behav. Brain Res. 314, 190-198 (2016).

63. Olexova, L., Stefanik, P. \& Krskova, L. Increased anxiety-like behaviour and altered GABAergic system in the amygdala and cerebellum of VPA rats - An animal model of autism. Neurosci. Lett. 629, 9-14 (2016).

64. Labouesse, M. A., Dong, E., Grayson, D. R., Guidotti, A. \& Meyer, U. Maternal immune activation induces GAD1 and GAD2 promoter remodeling in the offspring prefrontal cortex. Epigenetics 10, 1143-1155 (2015).

65. Richetto, J., Calabrese, F., Meyer, U. \& Riva, M. A. Prenatal versus postnatal maternal factors in the development of infection-induced working memory impairments in mice. Brain Behav. Immun. 33, 190-200 (2013).

66. Blatt, G. J. \& Fatemi, S. H. Alterations in GABAergic biomarkers in the autism brain: research findings and clinical implications. Anat. Rec. (Hoboken). 294 1646-1652 (2011).

67. Yip, J., Soghomonian, J. J. \& Blatt, G. J. Decreased GAD65 mRNA levels in select subpopulations of neurons in the cerebellar dentate nuclei in autism: an in situ hybridization study. Autism Res. 2, 50-59 (2009).

68. Zalewski, N. L., Lennon, V. A., Lachance, D. H., Klein, C. J., Pittock, S. J. \& McKeon A. P/Q- and N-type calcium-channel antibodies: Oncological, neurological, and serological accompaniments. Muscle Nerve 54, 220-227 (2016).

69. Spiciarich, M. C. \& Moshe, S. L. Voltage-gated P/Q-type calcium channel antibodies associated with cerebellar degeneration. Pediatr. Neurol. 62, 43-46 (2016).

70. Breitenkamp, A. F., Matthes, J. \& Herzig, S. Voltage-gated calcium channels and autism spectrum disorders. Curr. Mol. Pharmacol. 8, 123-132 (2015).

71. Gupta, S., Samra, D. \& Agrawal, S. Adaptive and innate immune responses in autism: rationale for therapeutic use of intravenous immunoglobulin. J. Clin. Immunol. 30, S90-S96 (2010). 